\title{
The polycystin-1 C-terminal fragment triggers branching morphogenesis and migration of tubular kidney epithelial cells
}

\author{
Christian Nickel, ${ }^{1}$ Thomas Benzing, ${ }^{2}$ Lorenz Sellin, ${ }^{2}$ Peter Gerke, ${ }^{2}$ Anil Karihaloo, ${ }^{1}$ \\ Zhen-Xiang Liu, ${ }^{1}$ Lloyd G. Cantley, ${ }^{1}$ and Gerd Walz ${ }^{2}$
}

${ }^{1}$ Section of Nephrology, Department of Medicine, Yale University, New Haven, Connecticut, USA
${ }^{2}$ Renal Division, University Hospital Freiburg, Freiburg, Germany

Address correspondence to: Lloyd G. Cantley, Section of Nephrology, Yale University, School of Medicine, P.O. Box 209029, New Haven, Connecticut 06529-8029, USA.

Phone: (203) 785-4186; Fax: (203) 785-4904; E-mail: lloyd.cantley@yale.edu.

Christian Nickel and Thomas Benzing contributed equally to this work.

The laboratories of Lloyd G. Cantley and Gerd Walz contributed equally to this work.

Received for publication April 2, 2001, and accepted in revised form December 28, 2001.

\begin{abstract}
Mutations of either PKD1 or PKD2 cause autosomal dominant polycystic kidney disease, a syndrome characterized by extensive formation of renal cysts and progressive renal failure. Homozygous deletion of $P k d 1$ or $P k d 2$, the genes encoding polycystin-1 and polycystin-2, disrupt normal renal tubular differentiation in mice but do not affect the early steps of renal development. Here, we show that expression of the C-terminal 112 amino acids of human polycystin-1 triggers branching morphogenesis and migration of inner medullary collecting duct (IMCD) cells, and support in vitro tubule formation. The integrity of the polycystin-2-binding region is necessary but not sufficient to induce branching of IMCD cells. The C-terminal domain of polycystin-1 stimulated protein kinase C- $\alpha$ (PKC- $\alpha)$, but not the extracellular signal-regulated kinases ERK1 or ERK2. Accordingly, inhibition of PKC, but not ERK, prevented polycystin-1-mediated IMCD cell morphogenesis. In contrast, HGF-mediated morphogenesis required ERK activation but was not dependent on PKC. Our findings demonstrate that the C-terminal domain of polycystin-1, acting in a ligand-independent fashion, triggers unique signaling pathways for morphogenesis, and likely plays a central role in polycystin- 1 function.
\end{abstract}

J. Clin. Invest. 109:481-489 (2002). DOI:10.1172/JCI200212867.

\section{Introduction}

Autosomal dominant polycystic kidney disease (ADPKD) is a common hereditary disease characterized by the development of gradually enlarging renal epithelial cysts that progressively impair renal function (recently reviewed in refs. 1-3). Most cases of ADPKD are caused by mutations of $P K D 1$, a gene that encodes polycystin-1. Polycystin-1 is a large integral membrane protein with several domains that suggest a role in cellcell or cell-matrix interactions $(4,5)$. It contains several transmembrane domains and a short intracellular C-terminal tail. The C-terminal polycystin-1 domain activates protein kinase C- $\alpha$ (PKC- $\alpha$ ) and the c-Jun N-terminal protein kinase (JNK1) (6), and triggers the accumulation of cytoplasmic $\beta$-catenin (7), indicating that this domain may play a crucial role in the signaling of polycystin-1. Mutations in a second gene, $P K D 2$, account for approximately $10-15 \%$ of all ADPKD cases (8). The disease is milder with this mutation than with the PKD1 mutation, and onset of end-stage renal disease occurs at a later age, but the phenotypic changes are otherwise similar. Since polycystin- 1 and polycystin- 2 interact via their C-terminal domains $(9,10)$, it has been postulated that both proteins participate in a common signaling pathway. Polycystin-2 is an integral membrane protein, with six transmembrane domains, that mediates nonselective cation currents in the presence of polycystin-1 (11). Both $P k d 1$ and $P k d 2$ gene deletions in mice result in embryonal or perinatal lethality, with cysts that originate in the second half of embryogenesis (12-16). These findings indicate that the polycystins are not required for mesenchymal-to-epithelial conversion and cell fate determination, but rather play a role in later stages of tubular epithelial cell differentiation and maturation. Recently, expression of polycystin-1 in Madin Darby canine kidney (MDCK) cells was shown to trigger the spontaneous formation of tubules (17). Based on our observation that the C-terminal domain of polycystin-1 can induce cellular activation and interaction with polycystin-2, we predicted that this region of polycystin- 1 is critical for initiating morphogenesis of renal epithelial cells. We adopted a highly efficient retroviral gene transfer approach to express the cytoplasmic domain of polycystin-1 in inner medullary collecting duct (IMCD) cells, and demonstrate here that the C-terminal 112 amino acids of human polycystin-1 trigger spontaneous branching morphogenesis and migration of renal tubular epithelial cells. 


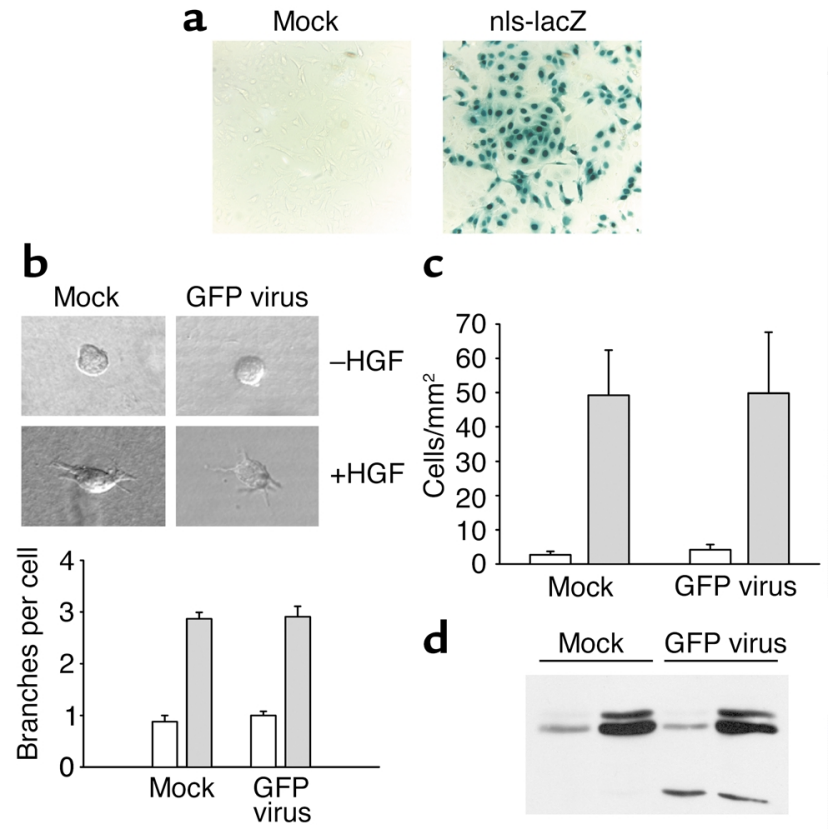

Figure 1

Retroviral gene transfer does not influence branching morphogenesis, migration, or MAP kinase activation of IMCD cells. (a) IMCD cells were transduced with a retrovirus directing the expression of $\beta$-galactosidase with a nuclear localization signal (nls-lacZ; right panel). Infected cells (right panel) and untransduced cells (left panel) were stained with $X$-GAL. Three repeated infections resulted in nearly $100 \%$ positive cells, without further selection. (b) Branching morphogenesis of infected IMCD cells. The figure shows phase-contrast microscopy of mocktreated and infected cells. Statistical evaluation demonstrates that retroviral transduction does not alter the branching behavior of IMCD cells (three independent experiments). White bars, basal condition; gray bars, HGF stimulation. (c) Migration of infected IMCD cells. Mock-treated and retrovirally transduced IMCD cells were subjected to cell migration assays as described. Neither basal cell migratory rates nor HGF-stimulated cell migration was altered by stable retroviral transduction in three independent experiments. White bars, basal condition; gray bars, HGF stimulation. (d) MAP kinase activation in infected IMCD cells. After serum starvation, mock-treated and retrovirally transduced IMCD cells were stimulated with solvent or HGF, and harvested. Equal amounts of protein were separated by $12 \%$ SDS-PAGE and immunoblotted with antibody against phosphorylated ERK1/2. Retroviral transduction does not activate ERK1 or ERK2 and does not influence HGF-stimulated activation.

\section{Methods}

Plasmids and viral vector construction. The CD16-, CD7-, and PKD1-containing plasmids used to generate the retroviral transfer vectors have been described $(6,9)$. The plasmid pLXSN was kindly provided by D. Miller (Fred Hutchinson Cancer Research Center, Seattle, Washington, USA). The plasmids pMMP.IRES.gfp, pMD-G, and pMD-gp were a kind gift of R. Mulligan (Harvard Medical School, Boston, Massachusetts, USA). The retroviral transfer vectors pLXSN-CD16.7, -CD16.7.PKD1, -CD16.7.IRES.gfp, and -CD16.7.PDK1.IRES.gfp were generated using standard cloning techniques. CD16.7 denotes the extracellular domain of CD16 and the transmembrane domain of CD7; PKD1 denotes the C-terminal cytoplasmic domain of human polycystin-1 (amino acids 4077-4302). In a subset of experiments, the cytoplasmic domain of polycystin- 1 was replaced by either the C-terminal 112 amino acids of polycystin-1 (amino acids 4191-4302) or a patient-derived truncation of the cytoplasmic domain at acid 4227 (amino acids 4077-4227). Point mutations of the C-terminal polycystin- 1 were generated by standard techniques.

Cell culture, virus production, and transduction. Immortalized mIMCD-3 cells (referred to as IMCD cells) (18) were grown in DMEM-F12 media supplemented with $10 \%$ FCS. The morphogenic response of these cells to EGF and HGF has been described $(19,20)$. The retroviral vector was produced by cotransfection of HEK $293 \mathrm{~T}$ cells with three plasmids ( $2.5 \mu \mathrm{g}$ of pMD-G, $7.5 \mu \mathrm{g}$ of $\mathrm{pMD}$-gp, and $10 \mu \mathrm{g}$ of the retroviral transfer vector) using the calcium phosphate method. The supernatant was harvested, centrifuged to remove cellular debris, and filtered. The titer was determined by FACS analysis of serial dilutions of the virus-containing stock solution. Cells were infected in the presence of $8 \mu \mathrm{g} / \mathrm{ml}$ polybrene for 4 hours, and in some experiments were selected 3-4 days after viral transduction in neomycin $(500 \mu \mathrm{g} / \mathrm{ml})$ to achieve $100 \%$ positive cells. Virus transduction and transgene expression were monitored by fluorescent microscopy of the co-expressed GFP protein and Western blot analysis.

Migration, branching process formation, and tubulogenesis assays. Migration assays were performed using a modified Boyden chamber assay as previously described (20). For branching process formation assays, cells were plated in a three-dimensional matrix that was a mixture of rat-tail type I collagen (Upstate Biotechnology, Lake Placid, New York, USA), 10× DMEM, and $200 \mathrm{mM}$ HEPES, pH 7.8 (8:1:1), and kept on ice, as previously described (21). IMCD cells were suspended at $1.5 \times 10^{5}$ cells $/ \mathrm{ml}$ of the collagen mixture, and dispensed into a flat-bottomed 96-well-plate. After 10-15 minutes at $37^{\circ} \mathrm{C}$, the polymerized collagen solution was overlaid with $100 \mu \mathrm{l}$ of the culture media with $0.5-1 \% \mathrm{FBS}$, with or without $40 \mathrm{ng} / \mathrm{ml} \mathrm{HGF}$ (R\&D Systems Inc., Minneapolis, Minnesota, USA). Plates were maintained at $37^{\circ} \mathrm{C}$ in a $\mathrm{CO}_{2}$ incubator for 24 hours, and then observed and photographed. Quantification of branching process formation was performed by counting 40 randomly selected individual cells per well, and dividing the number of branches by the total number of cells counted. The formation of multicellular structures (in vitro tubulogenesis) was monitored in a matrix containing Matrigel (Becton Dickinson and Co., Franklin Lakes, New Jersey, USA) and type I collagen. Plates were maintained for up to 7 days. The reagents staurosporine, calphostin $\mathrm{C}$, bis-indolylmaleimide I, and bis-indolylmaleimide $\mathrm{V}$ (Calbiochem-Novabiochem Corp., San Diego, California, USA) were used at the concentrations indicated. The MEK1 and MEK2 inhibitor U0126 (Promega, Madison, Wisconsin, USA) was used at a concentration of $10 \mu \mathrm{M}$. Each experiment was repeated at least three times. Statistical significance was determined using the Student $t$ test. 
a

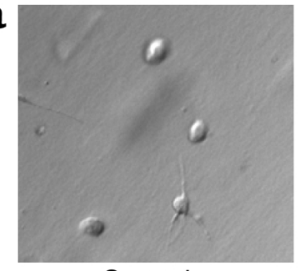

Control

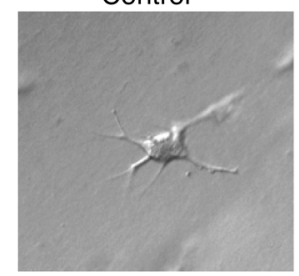

b

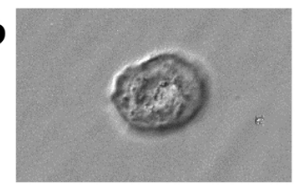

Control

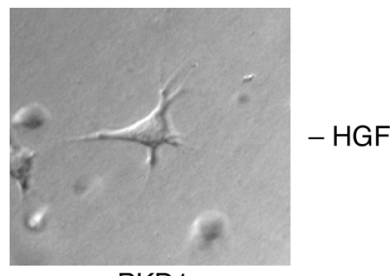

PKD1
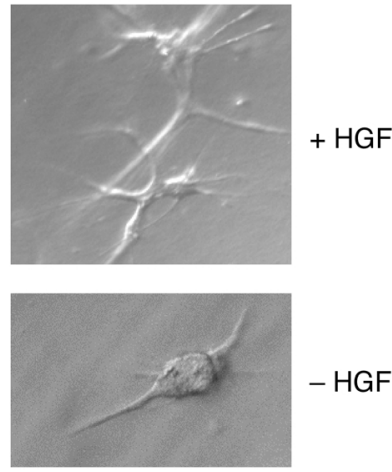

PKD1

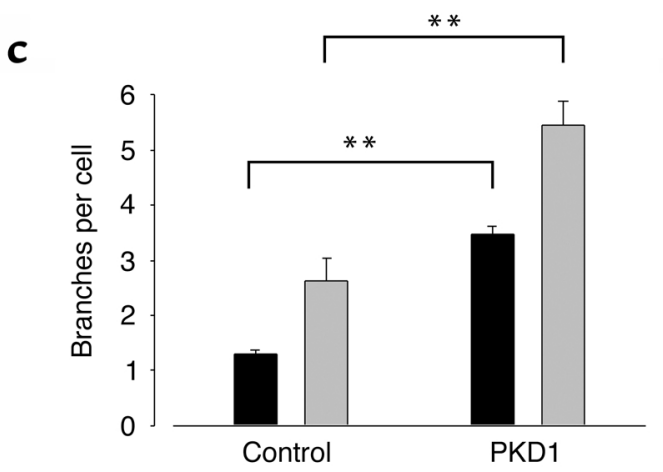

Western blot analysis. Western blot analysis and immunoprecipitation were performed as recently described $(6,22)$. Dually phosphorylated ERK1/2 was visualized with phosphospecific antiserum (New England Biolabs Inc., Beverly, Massachusetts, USA), while total amounts of Pyk2 and ERK1 were detected with activation-independent antisera (New England Biolabs Inc.). Equal loading was confirmed by amido black staining. The polycystin-2-specific antiserum has been recently described (23). A phospho-PKC (pan) antiserum (New England Biolabs Inc.) was utilized to detect activated PKC isoforms (PKC- $\alpha,-\beta_{1}$, $-\beta_{2},-\varepsilon,-\eta$, and $\left.-\delta\right)$.

\section{Figure 3}

Polycystin-induced branching does not require activation of the ERK1/2 pathway. (a) CD16.7-positive IMCD cells (control) and CD16.7.PKD1-positive IMCD cells (PKD1) were treated with either the specific MEK inhibitor U0126 or solvent, and subjected to branching morphogenesis assays. U0126 blocks the HGF-stimulated response, but has only a modest effect on polycystin-induced branching. (b) Polycystin-1 does not influence basal or HGF-mediated ERK1/2 phosphorylation. After stimulation with solvent or HGF, cells were harvested, and equal amounts of protein were separated by $12 \%$ SDS-PAGE and immunoblotted with antibody against phosphorylated ERK1/2.

\section{Figure 2}

Expression of the membrane-anchored C-terminal cytoplasmic domain of polycystin-1 induces branching morphogenesis in IMCD cells. (a) CD16.7-positive (control) and CD16.7.PKD1-positive IMCD cells (PKD1) were subjected to branching morphogenesis assays. All cells were positive for the transgene, and expressed the CD16.7 fusion protein at the plasma membrane, as demonstrated by immunofluorescence microscopy of unfixed, nonpermeabilized cells. Expression of the cytoplasmic tail of polycystin-1 induces branching and augments the HGF-stimulated response. (b) IMCD cells cultured for 48 hours in Matrigel in the absence of HGF display a branching, elongated phenotype, as compared to the cystic phenotype seen in control cells. (c) Evaluation of three independent experiments demonstrates the highly significant effect of polycystin-1 on both basal and HGF-stimulated branching morphogenesis. Black bars, basal condition; gray bars, HGF-stimulated IMCD cells. ${ }^{*} P<0.01$.

In vitro kinase assay. The cellular activity of PKC- $\alpha$ was determined by an in vitro kinase assay as previously described (23). Briefly, HEK 293T cells were lysed 24 hours after transfection with PKC- $\alpha$ and expression plasmids as indicated. PKC- $\alpha$ was immunoprecipitated using a monoclonal antibody against PKC- $\alpha$ (Transduction Laboratories, Lexington, Kentucky, USA). Immobilized PKC- $\alpha$ was then incubated with neurogranin (as an exogenous substrate) and $5 \mu \mathrm{Ci}$ of $\left[\gamma^{-32} \mathrm{P}\right] \mathrm{ATP}$. The incorporated radioactivity was determined, after two washes with $75 \mathrm{mM}$ phosphoric acid, by liquid scintillation spectrophotometry with a phosphocellulose membrane (Pierce Chemical Co., Rockford, Illinois, USA).

\section{Results}

Retroviral gene transfer does not alter the biological properties of IMCD cells. Two immortalized renal epithelial cell lines, MDCK and IMCD, have been widely used to study tubular kidney epithelial morphogenesis in vitro. Since clonal selection of these cells can result in phenotypic changes not related to the expressed transgene, we chose to adopt a highly efficient retroviral gene transfer to manipulate IMCD cells (24). To monitor transduction efficiencies, the cDNA of enhanced green

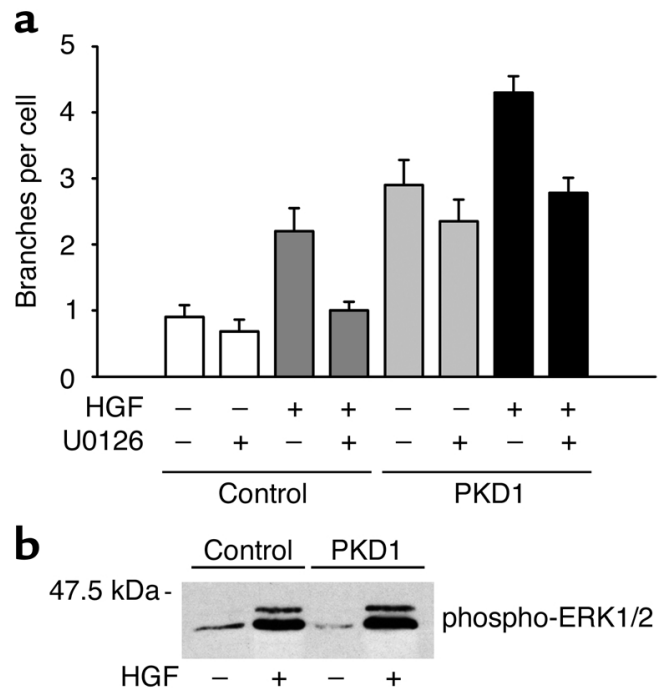




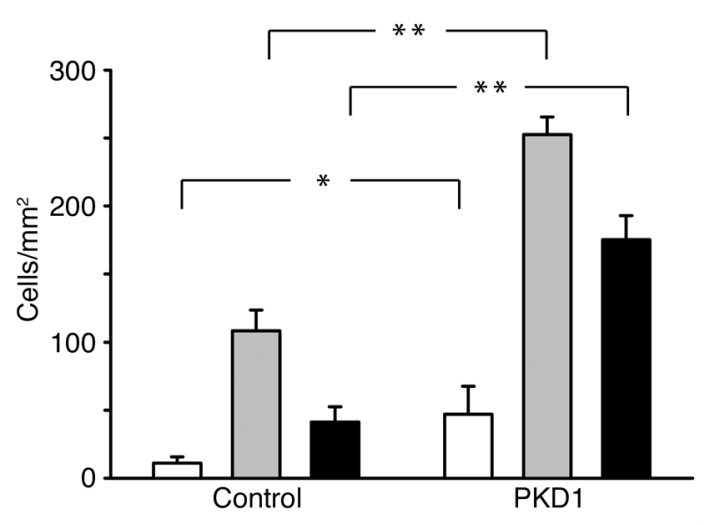

fluorescent protein (GFP) was inserted downstream of an internal ribosome entry site (IRES), resulting in the simultaneous expression of GFP and the transgene of interest. Figure 1a demonstrates that after two to three repetitive retroviral infections, nearly all IMCD cells express $\beta$-galactosidase. To avoid multiple integration events, cells were infected at a low multiplicity of infection (two to five). Approximately $60-80 \%$ of cells were found to be GFP-positive at this multiplicity of infection; virtually all cells became GFP-positive after a brief selection in neomycin. Since the integration site of the transgene in the host chromosome after viral transduction is nearly random, each GFP-positive, neomycin-resistant cell represents an independent integration event.

As demonstrated in Figure 1b, transduction of IMCD cells did not influence branching morphogenesis, or alter the migratory response of tubular epithelial cells to chemotactic stimuli (Figure 1c). In addition, retroviral infection did not affect mitogen-activated protein (MAP) kinase signaling, as shown for the HGF-induced activation of the MAP kinases ERK1 and ERK2 (Figure 1d). Thus, this retroviral gene transfer approach allows highly efficient expression of transgenes without detectable effects on branching morphogenesis, migration, or MAP kinase signaling in IMCD cells.

The C-terminal domain of polycystin-1 triggers spontaneous branching morphogenesis. We have previously shown that a membrane-anchored C-terminus of polycystin- 1 activates signaling pathways such as PKC- $\alpha$ and JNK1 in HEK 293T cells (6). The recent finding that wild-type

\footnotetext{
Figure 5

The polycystin-1 effect on branching morphogenesis and migration can be mimicked by a short segment (amino acids 4191-4302) of the cytoplasmic domain of human polycystin-1. (a) IMCD cells were transduced with retroviral vectors encoding either CD16.7 (white bars), CD16.7.PKD1 (full-length cytoplasmic domain of polycystin1, gray bars) or CD16.7.PKD14191-4302 (black bars), and subjected to branching morphogenesis assays. Expression of the C-terminal 112 amino acids of polycystin-1 resulted in increased basal and HGFstimulated branching morphogenesis that was indistinguishable from that observed with the full-length cytoplasmic domain. **P< $0.01 ; n=3$. (b) Expression of the C-terminal 112 amino acids of polycystin-1 initiate IMCD cell migration and augment HGF-stimulated migration. ${ }^{*} P<0.01 ; n=3$.
}

\section{Figure 4}

Expression of the membrane-anchored C-terminal cytoplasmic domain of polycystin-1 initiates undirected migration in IMCD cells. IMCD cells were plated on polycarbonate filters and subjected to a migration assay as described in Methods, using a modified Boyden chamber. Cells expressing PKD1 1077-4302 were found to have increased basal migratory rates, as well as an exaggerated response to a chemoattractant gradient of HGF. The MEK inhibitor U0126 significantly inhibited the HGF-stimulated migration in control cells, but only partially prevented the polycystin-induced migratory response $(n=3) .{ }^{*} P<0.05,{ }^{*} P<0.01$. White bars, basal condition; gray bars, HGF-stimulated; black bars, HGF plus U0126.

polycystin-1 triggers branching morphogenesis in MDCK cells (17) prompted us to examine the question of whether the C-terminal cytoplasmic domain of polycystin-1 is sufficient to trigger complex biological programs. IMCD cells were transduced with either a CD16.7- or CD16.7.PKD1-expressing retrovirus, and briefly selected in neomycin to obtain a pool of $100 \%$ positive cells. When wild-type IMCD cells are cultured in type I collagen in the absence of HGF, these cells form few processes, and fail to develop into tubules (19). In the presence of HGF or EGF, these cells form elongated branching processes and proliferate to form branching tubular structures. Expression of the membraneanchored cytoplasmic tail of polycystin-1 triggered branching morphogenesis in these tubular kidney cells in the absence of an additional stimulus (Figure 2a). This effect could also be demonstrated in a matrix (Matrigel) that usually induces cyst development in vitro (Figure 2b), and was similarly observed with MDCK cells (data not shown). In response to treatment with HGF, CD16.7-expressing control cells showed the expected increase in branching

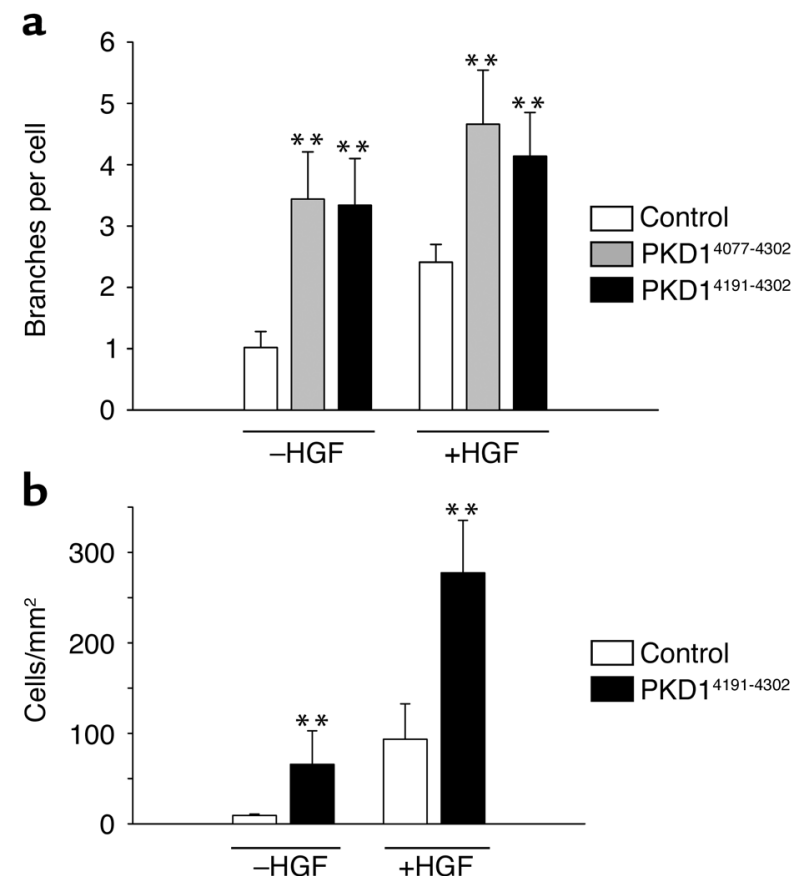


morphogenesis (Figure 2a). In cells expressing the cytoplasmic domain of polycystin-1, basal branching morphogenesis was equivalent to the maximal degree of branching seen in response to HGF. Interestingly, stimulation of the polycystin-1-expressing cells with HGF resulted in a further augmentation of the branching phenotype (Figure 2c), suggesting that polycystin-1 activates signaling pathways distinct from those that are critical for HGF-mediated morphogenesis. Since we have recently demonstrated that HGF requires activation of the ERK1/2 MAP kinase pathway for branching morphogenesis and migration (25), we examined the effects of inhibition of MEK1/2 in polycystin-1-mediated branching morphogenesis. As previously reported for native IMCD cells (25), treatment of control cells expressing CD16.7 with the MEK-specific inhibitor U0126 completely prevented HGF-dependent branching morphogenesis (Figure 3a). In contrast, branching induced by the expression of membrane-tagged polycystin-1 was only modestly diminished. In polycystin-1-expressing cells stimulated with HGF, the component of increased branching attributable to the HGF stimulation was abolished by treatment with U0126, whereas the basal increase in branching due to polycystin-1 expression was not altered. Western blot analysis revealed that ERK1 and 2 were not activated in IMCD cells expressing the C-terminal domain of polycystin-1, nor did polycystin-1 influence the HGF-stimulated ERK1/2 activation (Figure $3 b$ ).

Polycystin-1 triggers spontaneous migration of IMCD cells. It has been postulated that tubulogenesis requires cell migration and cell proliferation prior to the formation of lumen-containing tubules (26). We tested whether the C-terminal domain of polycystin-1 mediates growth factor-independent cell migration. As demonstrated in Figure 4, IMCD cells expressing the C-terminal domain of polycystin-1 displayed an increase in undirected migration, and significantly exaggerated the response to the HGF-mediated chemotactic stimulus. The MEK inhibitor U0126 markedly inhibited the HGF-stimulated migration in control cells, but had only a modest inhibitory effect on the polycystin-induced migratory response. Taken together, these findings suggest that polycystin-1 triggers migration of IMCD cells through a largely ERK1/2-independent pathway.

The C-terminal 112 amino acids of polycystin-1 mediate branching and migration. It has been shown that the direct interaction between polycystin- 1 and polycystin2 involves the coiled-coil domain contained within the C-terminus of the polycystin-1 protein $(9,10)$. In addition, this region triggers recently described intracellular signaling pathways $(6,7)$, leading us to hypothesize that the specific protein interactions mediated by the coiled-coil domain of polycystin-1 might be responsible for the induction of branching morphogenesis and migration. A truncated construct encoding only the C-terminal 112 amino acids of polycystin-1, but still including the entire coiled-coil region, was generated to examine this possibility. As shown in Figure 5, the
112 amino acids of the membrane-anchored C-terminal of polycystin-1 mediated branching morphogenesis and migration in a manner that was indistinguishable from that mediated by the full-length cytoplasmic domain, suggesting that this part of the cytoplasmic tail of polycystin-1 serves as a ligand-independent, constitutively active form of polycystin-1.

Truncation of the C-terminal domain of polycystin-1 prevents in vitro tubulogenesis. To demonstrate that short-term branching of IMCD cells expressing the C-terminal domain of polycystin- 1 is closely linked to the formation of lumen-containing tubules, we performed long-term tubulogenesis assays in IMCD cells expressing either full-length C-terminal polycystin-1 (CD16.7.PKD1), or a naturally occurring truncation mutation of the C-terminus that has been detected in several patients with PKD (CD16.7.R4227X). In a Matrigel/type I collagen matrix, IMCD cells expressing the full-length C-terminal domain of polycystin-1 underwent extensive branching and elongation during the first several days of culture, resulting in the formation of multicellular branching linear structures by day 4 (Figure 6). These structures further developed into tubules with central lumens, beginning on culture day 5 . In contrast, expression of the R4227X truncation mutant of polycystin-1 failed to support branching during the initial days of

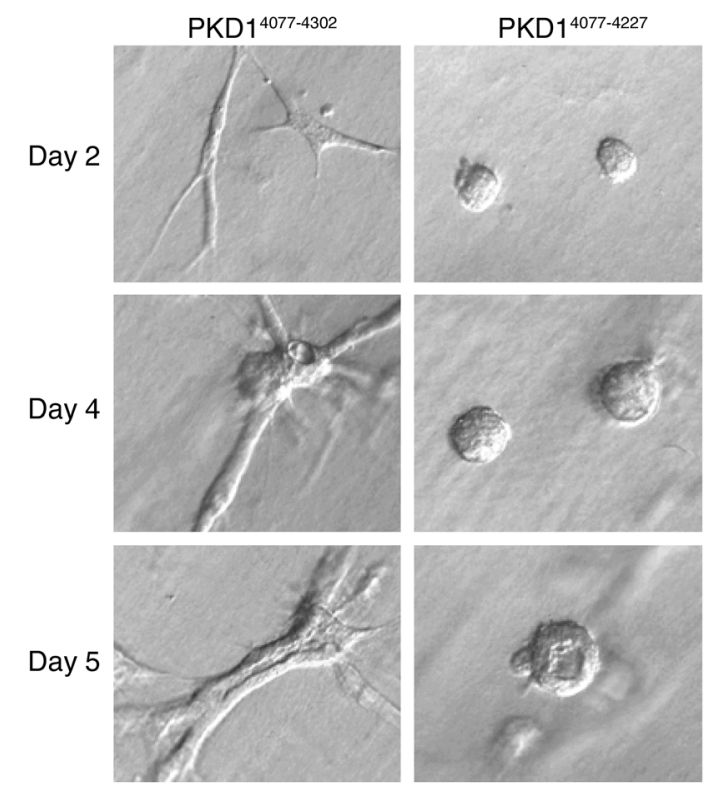

\section{Figure 6}

IMCD cells expressing the C-terminal domain of polycystin-1 form multicellular tubular structures. IMCD cells expressing either CD16.7.PKD1 (PKD ${ }^{4077-4302}$ ) or the patient-derived truncation mutation CD16.7.R4227X (PKD 4077-4227) were cultured for 5 days in a Matrigel/type I collagen mixture. Single-cell branching by cells expressing CD16.7.PKD1 on culture days 1 and 2 correlated with the formation of branching tubular structures by days 4 and 5. Expression of the CD16.7.R4227X truncation mutant did not support branching on days 1 and 2, and resulted in the formation of multicellular spherical structures by days 4 and 5 . The development of lumens in both types of structures began by day 5 of culture. Magnification of images, $\times 20$; representative structures are shown. 
a

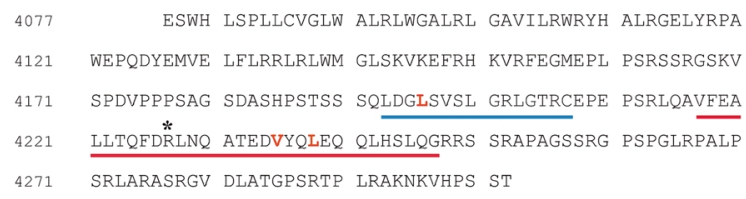

b

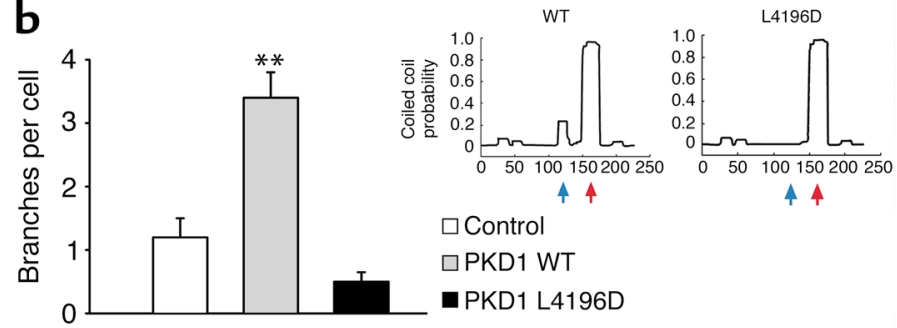

c

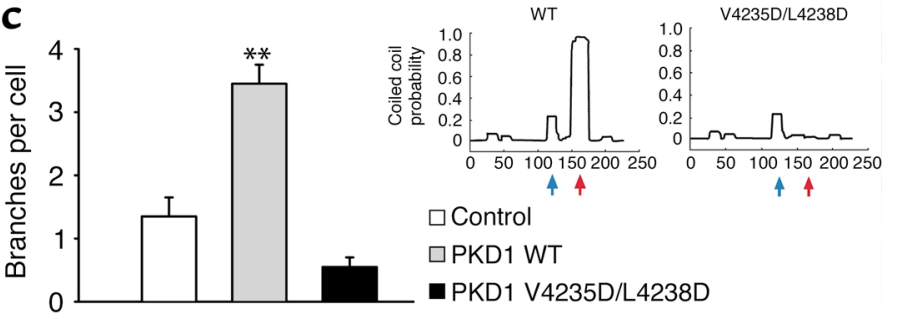

d

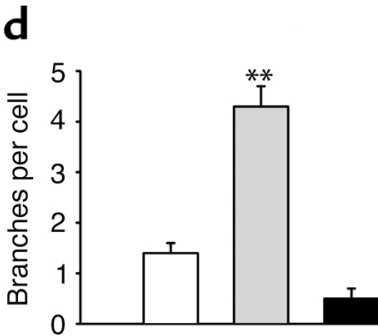

ब
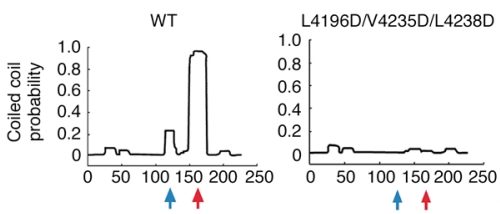

$\square$ Control

$\square$ PKD1 WT

PKD1 L4196D/N4235D/L4238D culture, and ultimately resulted in the formation of multicellular spherical structures, with or without lumens, by culture days 4 and 5 . Thus, loss of the C-terminal 75 amino acids of polycystin- 1 abrogated the initiation of branching morphogenesis, and ultimately prevented tubulogenesis in these cells.

The integrity of the polycystin-2-binding domain is necessary, but not sufficient, to trigger branching morphogenesis. It has been postulated that polycystin-1 and polycystin2 participate in a common signaling cascade that prevents the formation of renal tubular cysts during embryogenesis $(9,10)$. To test the hypothesis that branching morphogenesis triggered by polycystin-1 requires interaction with polycystin-2, we mutated the polycystin-2-binding domain of polycystin-1. The polycystin-2-binding region has been mapped to the coiled-coil domain of polycystin-1 $(9,10)$, which contains five heptad repeats (COILS version 2.1, window 28;http://www.ch.embnet.org/software/COILS_form. html) (10). This domain of polycystin- 1 is preceded by two additional heptad repeats and a proline-rich linker region. Using the Lupas coiled-coils prediction at low stringency (27) (COILS version 2.1, window 14), we introduced single amino acid substitutions to independently disrupt the upper two heptad repeats and

\section{Figure 7}

Mutational analysis reveals that the integrity of two distinct regions of the cytoplasmic domain of human polycystin- 1 is necessary to trigger branching morphogenesis. (a) Depicted are the two $\mathrm{N}$-terminal heptad repeats (blue line, blue arrow) and the five $\mathrm{C}$-terminal heptad repeats (red line, red arrow), intersected by the proline-rich region of the C-terminus of polycystin-1. The mutated amino acids (L4196, V4235, and L4238) are shown in red text. The position of the patient truncation mutant R4227X is marked with a single asterisk. (b-d) IMCD cells were transduced with a retrovirus expressing CD16.7 fused to the cytoplasmic tail of polycystin-1 (PKD1 WT), or to three mutations (PKD1 L4196D), the double mutation (PKD1 V4235D/L4238D), and the triple mutation (PKD1 L4196D/V4235D/L4238D). A retrovirus expressing CD16.7 was used as a control. The coiled-coils prediction reveals that the $L 4196 \mathrm{D}$ mutation dramatically reduces the coiled-coils probability for the $\mathrm{N}$-terminal heptad repeats (b), and the V4235D/L4238D double mutation abolishes the coiled-coils probability for the C-terminal heptad repeats (c). Accordingly, the triple mutation L4196D/V4235D/L4238D virtually eliminates the coiled-coils probability for the entire region (d). All three mutations completely abrogate branching morphogenesis of IMCD cells. ${ }^{*} P<0.01$.

the coiled-coil structure. Although all constructs were correctly targeted to the plasma membrane (data not shown), disruption of either the upper two heptad repeats mutant PKD1 L4196D) or the coiled-coil structure (mutant PKD1 V4235D/L4238D) completely abrogated polycystin-1-mediated branching of IMCD cells (Figure 7). Similarly, the R4227X truncation mutation of polycystin-1, which eliminates the coiled-coil structure, abolished the ability of polycystin- 1 to induce branching of IMCD cells (Figure 8). In contrast, binding of polycystin-2 was prevented by disruption of the coiled-coil domain (Figure 9), but not by disruption of the upper two heptad repeats (Figure 9). These findings indicate that the integrity of all heptad repeats of polycystin-1 are required for polycystin-1-mediated branching of IMCD cells, whereas only the coiled-coil structure of polycystin-1 is essential for interaction with polycystin-2. It is interesting to note that the extent of branching of IMCD cells expressing the polycystin-1 mutants remained well below the level of branching of control cells. Since IMCD cells express endogenous polycystins (data not shown), the polycystin-1 mutants appear to exert a dominant negative effect on branching induced by endogenous proteins.

Branching morphogenesis by polycystin-1 is mediated by $P K C$. We have previously demonstrated that the C-terminal domain of polycystin- 1 activates PKC- $\alpha$ (6). To test whether morphogenesis requires activation of PKC, we examined the effects of the protein kinase inhibitor staurosporine and the nonselective PKC inhibitor calphostin $\mathrm{C}$ on branching morphogenesis and migration triggered by polycystin- 1 . As demonstrated in Figure 10a, polycystin-1-mediated branching was potently inhibited by either $100 \mathrm{pM}$ 


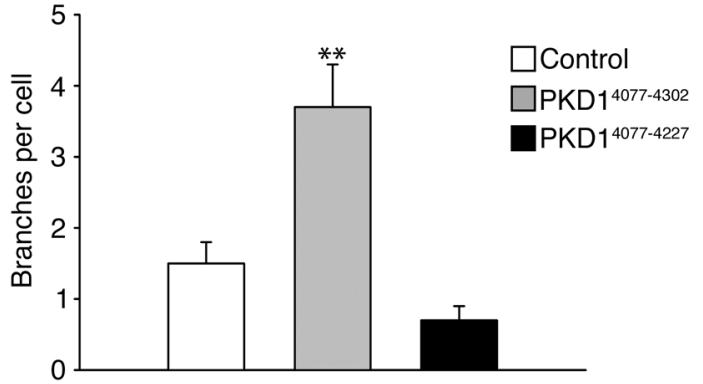

Figure 8

The R4227X polycystin-1 nonsense mutation of patients with ADPKD abrogates branching morphogenesis. IMCD cells were transduced with a retrovirus expressing CD16.7 fused to the cytoplasmic tail of polycystin-1 (PKD14077-4302), or to cytoplasmic polycystin-1 truncated at R4227 (PKD1 4077-4227). A retrovirus expressing CD16.7 was used as a control. Truncation of the cytoplasmic tail of polycystin-1 at R4227 abrogated branching morphogenesis of IMCD cells. ${ }^{*} P<0.01$.

staurosporine or $100 \mathrm{nM}$ calphostin C. Furthermore, the PKC inhibitor bis-indolylmaleimide I inhibited branching of IMCD cells expressing the C-terminal domain of polycystin-1, whereas its inactive isomer bisindolylmaleimide $\mathrm{V}$ had no detectable effect (Figure 10a). Finally, Gö6976, a PKC inhibitor selective for the calcium-dependent PKC isoforms $\alpha, \beta$, and $\delta$ (28), blocked polycystin-1-mediated branching of IMCD cells in a dose-dependent fashion (Figure 10b). Similarly, inhibition with Gö6976 markedly diminished polycystin-1-dependent IMCD cell migration (Figure 10c); identical effects were obtained for staurosporine and calphostin C (data not shown).

To further explore our observation that HGF and polycystin-1 use different signaling pathways for their morphogenic responses, we compared the effects of inhibition of PKC- $\alpha$ on HGF- and polycystin-1-mediated branching morphogenesis. In contrast to the marked inhibition of branching morphogenesis that is seen in CD16.7.PKD1-expressing cells treated with Gö6976 (Figure 10d), inhibition of PKC- $\alpha$ failed to prevent HGF-stimulated IMCD cell branching (Figure $10 \mathrm{~d}$, compare columns 5 and 6). Stimulation of polycystin-1-expressing cells with HGF resulted in a further increase in branching that was inhibited back to the level of branching seen with polycystin-1 alone following Gö6976 treatment (Figure 10d). Since these results suggest that branching morphogenesis is closely linked to the polycystin-1-mediated activation of PKC, we postulated that the polycystin- 1 mutants that fail to mediate branching would lack the ability to activate PKC. Figure 11 demonstrates that the C-terminal domain of polycystin- 1 increased the activity of PKC- $\alpha$, while neither the two polycystin- 1 mutants (PKD1 L4196D and PKD1 V4235D/ L4238D), nor the patient-derived truncated form of polycystin-1, had any significant effect on the activity of PKC- $\alpha$. Increased endogenous PKC activity was detectable in IMCD cells expressing the C-terminal domain of polycystin-1 (Figure 11, b and c). Taken together, these results indicate that activation of PKC is an essential component of polycystin-1-mediated branching morphogenesis and migration.

\section{Discussion}

Normal renal development requires the presence of polycystin(s). Homozygous inactivation of either $P k d 1$ or $P k d 2$ results in the development of renal cysts and embryonal lethality $(12,14,16)$. The cysts first become apparent at embryonic day 15.5, a time when renal tubules are undergoing progressive lengthening and maturation $(12,16)$. Interestingly, the polycystins do not appear to affect the earliest steps of renal embryogenesis, since neither the repetitive branching of the ureteric bud, nor mesenchymal-to-epithelial conversion with the generation of new nephron precursors, appears to be affected by the absence of either polycystin $(12,14,16)$. Despite the apparently normal early tubule formation in $P \mathrm{KD}^{-/-}$mice, there are several lines of evidence suggesting a role for polycystin-1 in tubulogenesis. Incubation of ex vivo renal organ cultures with short peptides, homologous to the PKD repeats, blocked branching of the ureteric bud (29), while overexpression of wild-type polycystin-1 triggered spontaneous tubulogenesis in MDCK cells (17). Our findings confirm the observation that expression of polycystin-1 can support tubulogenesis in vitro, and demonstrate that the morphogenic

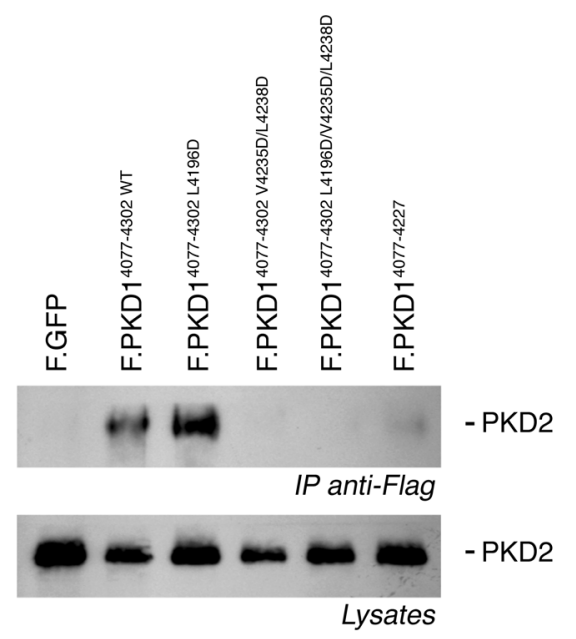

\section{Figure 9}

The polycystin-1 R4227X nonsense mutation and the V4235/L4238D mutation, but not the L4196D mutation, abolish binding of polycystin-2. Flag-tagged versions of the mutated cytoplasmic tail of polycystin- 1 were coexpressed with human wild-type polycystin-2 in HEK 293T cells. After immunoprecipitation of the Flag-tagged polycystin-1 proteins immobilized polycystin- 2 was detected using a polycystin-2-specific antiserum. While polycystin-2 binds to the wild-type cytoplasmic tail of polycystin- 1 and the L4196D mutation, it does not recognize the PKD1 V4235D/L4238D, the PKD1 L4196D/V4235D/L4238D, or the PKD1 R4227X mutations. Whole cell lysates (Lysates) were immunoblotted with anti-polycystin-2 to demonstrate equal amount of starting protein for each immunoprecipitation (IP anti-Flag). These findings demonstrate that the five $\mathrm{C}$-terminal heptad repeats, predicted to form a coiled-coil structure, but not the two $\mathrm{N}$-terminal heptad repeats, are essential for the interaction of polycystin-2 with polycystin-1. 
a
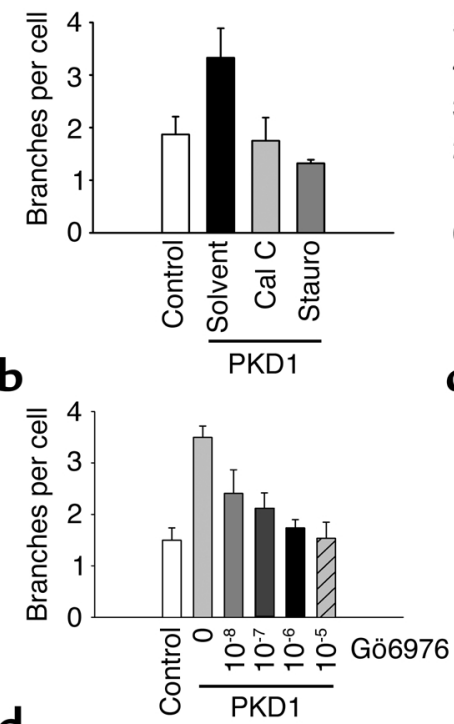

C
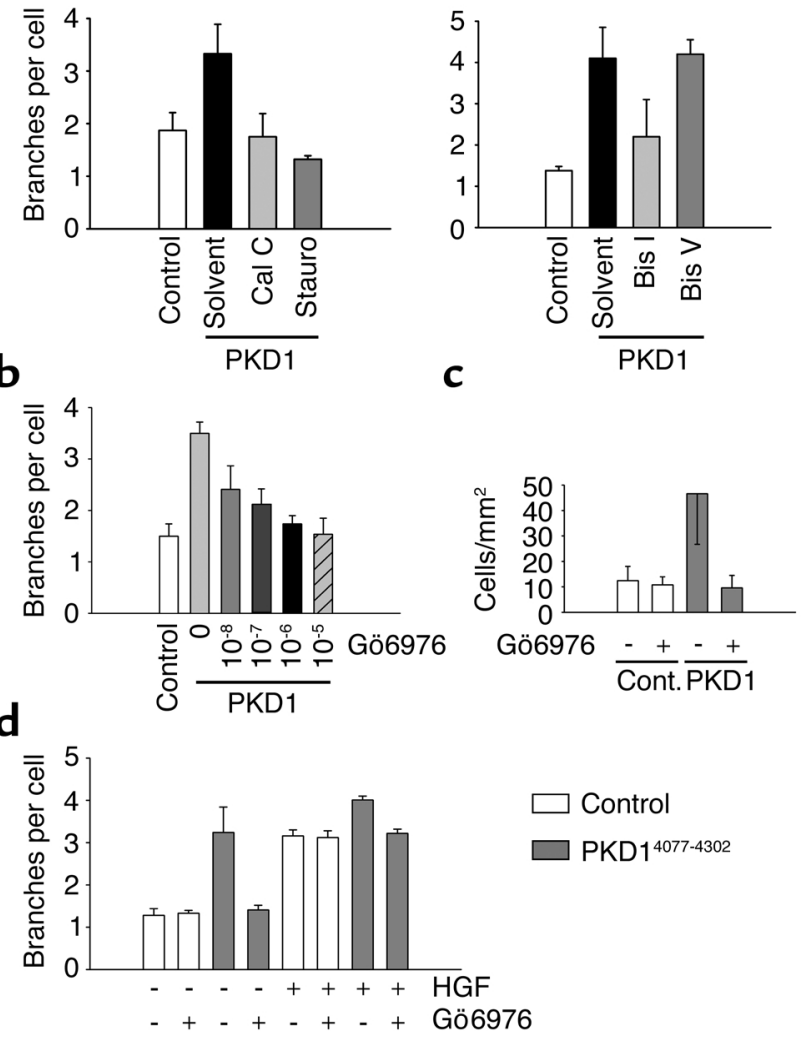

changes triggered by polycystin- 1 are mediated by the C-terminal domain. In fact, the C-terminal 112 amino acids of polycystin-1 are sufficient to initiate both branching morphogenesis and spontaneous migration of tubular epithelial cells, whereas loss of the integrity of the coiled-coil region prevents these events. Whether the ability of polycystin- 1 to promote morphogenic changes in tubular epithelial cells is important during developmental tubulogenesis (and is replaced by redundant pathways in the $P \mathrm{KD1}^{-/-}$mouse), or whether the signaling events directed by polycystin- 1 instead play a role in the maintenance of normal tubular architecture, remains to be determined.

\section{Figure 11}

Activation of protein kinase $C-\alpha$ requires the integrity of the heptad repeats of the C-terminal domain of polycystin-1. (a) HEK 293T cells were transfected with wild-type PKC- $\alpha$ and expression plasmids as indicated. The activity of immunoprecipitated PKC- $\alpha$ was determined by in vitro kinase assays. The $\mathrm{C}$-terminal domain of polycystin- 1 triggered a $270 \%$ activation of PKC- $\alpha$, while polycystin-1, containing the L4196D or V4235D/L4238D mutations, completely lost the ability to activate PKC- $\alpha$. (b) The C-terminal domain of polycystin- 1 activates endogenous PKC- $\alpha$ in IMCD cells. Endogenous PKC- $\alpha$ was immunoprecipitated using an isoform specific antibody, and subjected to in vitro kinase assays. The increase in PKC- $\alpha$ activity by the C-terminal domain of polycystin- 1 was comparable to the activation triggered by PMA ( $0.2 \mu \mathrm{M}$ for 15 minutes). (c) Activation of endogenous PKC isoforms by the C-terminal domain of polycystin- 1 was confirmed using a phospho-specific PKC antiserum. To control for loading, the Western blot was reprobed with antisera that detect the total amounts of the protein kinases ERK1 and Pyk2.

\section{Figure 10}

Polycystin-1-mediated branching morphogenesis and migration are abrogated by inhibition of PKC activity. (a) IMCD cells, transduced with a retrovirus expressing CD16.7.PKD1, were treated with the nonspecific PKC inhibitors staurosporine (Stauro; $100 \mathrm{pM}$ ) or calphostin C (Cal C; 100 nM) for 24 hours. Both inhibitors completely abrogated polycystin-1-mediated branching of IMCD cells $(n=3)$. The selective PKC inhibitor bis-indolylmaleimide I (Bis I, 5 $\mu \mathrm{M}$ ), but not the inactive isomer bis-indolylmaleimide $\mathrm{V}$ (Bis $\mathrm{V}, 5$ $\mu \mathrm{M})$, similarly inhibited polycystin-1-mediated branching of IMCD cells. (b) Gö6976, a selective inhibitor of the calcium-dependent $\alpha$, $\beta$, and $\delta$ PKC isoforms, abrogates polycystin-1-dependent branching morphogenesis in a dose-dependent fashion. (c) Gö6976 (1 $\mu \mathrm{M}$ ) inhibits polycystin-1-mediated migration. (d) IMCD cells expressing CD16.7 or CD16.7.PKD1 were treated with either Gö6976, HGF, or both reagents. Gö6976 (1 $\mu \mathrm{M})$ inhibited the polycystin-1-mediated branching, but had no effect on basal branching. Treatment with HGF increased IMCD cell branching, and had an additive effect on polycystin-1-mediated branching. Gö6976 failed to prevent HGF-mediated branching.

We have previously demonstrated that the C-terminal domain of polycystin-1, fused to the extracellular domain of CD16 and the transmembrane domain of CD7, mediates the activation of PKC- $\alpha$ and JNK1 (6), as well as the accumulation of cytoplasmic $\beta$-catenin (7). However, in contrast to the extensively studied epithelial morphogen HGF, the C-terminal domain of polycystin-1 does not activate the MAP kinases ERK1 or ERK2 (6). HGF is a secreted glycoprotein that stimulates the motility as well as the branching of several types of epithelial cells, including renal MDCK and IMCD cells. The receptor for HGF is the transmembrane tyrosine kinase $\mathrm{p} 190^{\mathrm{Mer}}$, encoded by the c-met proto-oncogene. Autophosphorylation of $\mathrm{p} 190^{\mathrm{Met}}$ in response to HGF triggers binding of several $\mathrm{SH} 2$ domain-containing proteins that are critical for its morphogenic actions, including phosphoinositide 3kinase, Grb2-associated binder 1 (GAB1), and phospholipase C- $\gamma$ (reviewed in ref. 30). In addition, the

a

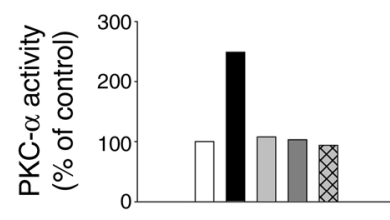

b

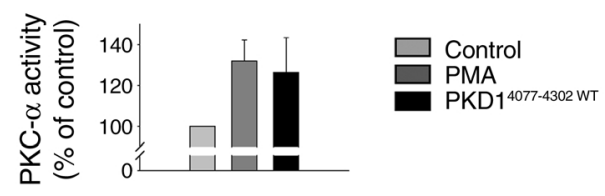

c

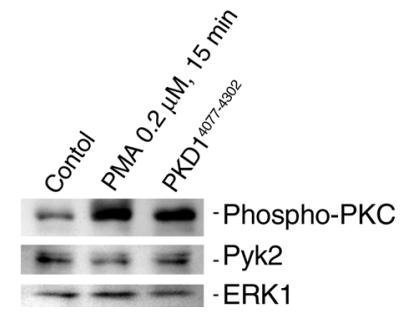


MAP kinases ERK1 and ERK2 were recently identified as essential components of HGF-mediated renal epithelial morphogenesis (25). In the present study, we demonstrate that polycystin-1-mediated morphogenesis is not dependent on MAPK activation, suggesting that polycystin-1 triggers branching through signaling pathways that are distinct from those activated by HGF. Since the C-terminal domain of polycystin-1 triggers activation of PKC- $\alpha$ (6), we delineated the role of PKC- $\alpha$ in polycystin-1-mediated branching morphogenesis and migration. Both nonselective PKC inhibitors and a PKC inhibitor that is specific for the calcium-dependent isoforms PKC- $\alpha$ and PKC- $\beta 1$, inhibited polycystin-1-mediated branching and migration, but failed to prevent HGF-mediated branching morphogenesis. Furthermore, polycystin- 1 mutations that failed to trigger branching lack the ability to activate PKC- $\alpha$, confirming that PKC- $\alpha$ plays a central role in polycystin-1-mediated morphogenic signaling.

The mutational analysis of polycystin- 1 revealed that the integrity of the polycystin-2-binding domain of polycystin-1, located within the coiled-coil structure, is necessary to mediate branching of IMCD cells. However, association of polycystin- 1 with polycystin- 2 is clearly not the only interaction that is required, since mutation of the two N-terminal heptad repeats, which did not affect the interaction of polycystin- 1 with polycystin-2, completely eliminated polycystin-1-mediated branching morphogenesis. These findings suggest that the interaction with polycystin-2 is necessary, but not sufficient, to mediate branching morphogenesis of IMCD cells. Of note, since the polycystin-1 L4196D point mutation preserves the coiled-coil domain, but abrogates branching, polycystin-1-mediated morphogenesis of IMCD cells is not simply the result of nonspecific recruitment of cellular proteins that bind to coiled-coil domains. Instead, we speculate that the assembly of a multimeric protein complex, mediated by distinct regions of the cytoplasmic tail of polycystin-1, is required to trigger branching morphogenesis of tubular epithelial cells.

Our findings have several important implications: (a) the C-terminal 112 amino acids of polycystin- 1 are sufficient to trigger ligand-independent migration and branching of IMCD cells; (b) polycystin-1-mediated branching and migration involve activation of PKC- $\alpha$, but not activation of ERK1 or ERK2; (c) the polycystin2-binding domain of polycystin-1 is necessary, but not sufficient, for branching morphogenesis; and (d) the heterologous expression of the C-terminal domain of polycystin-1 resembles wild-type polycystin-1 expression, and provides a simple model system to further delineate polycystin-1-mediated signaling.

\section{Acknowledgments}

We thank the members of the Cantley and Walz laboratories for helpful discussion. This work was supported by NIH grant DK-52897 (to L.G. Cantley and G. Walz) and Deutsche Forschungsgemeinschaft grants Wa 597 (to G. Walz) and Be 2212 (to T. Benzing).
1. Arnaout, M. 2001. Molecular genetics and pathogenesis of autosomal dominant polycystic kidney disease. Annu. Rev. Med. 52:93-123.

2. Calvet, J.P., and Grantham, J.J. 2001. The genetics and physiology of polycystic kidney disease. Semin. Nephrol. 21:107-123.

3. Somlo, S., and Markowitz, G.S. 2000. The pathogenesis of autosomal dominant polycystic kidney disease: an update. Curr. Opin. Nephrol. Hypertens. 9:385-394.

4. 1995. Polycystic kidney disease: the complete structure of the PKD1 gene and its protein. The International Polycystic Kidney Disease Consortium. Cell. 81:289-298.

5. Hughes, J., et al. 1995 . The polycystic kidney disease 1 (PKD1) gene encodes a novel protein with multiple cell recognition domains. Nat. Genet. 10:151-160.

6. Arnould, T., et al. 1998. The polycystic kidney disease 1 gene product mediates protein kinase $\mathrm{C}$ alpha-dependent and c-Jun $\mathrm{N}$-terminal kinase-dependent activation of the transcription factor AP-1. J. Biol. Chem. 273:6013-6018.

7. Kim, E., et al. 1999. The polycystic kidney disease 1 gene product modulates Wnt signaling. J. Biol. Chem. 274:4947-4953.

8. Mochizuki, T., et al. 1996. PKD2, a gene for polycystic kidney disease that encodes an integral membrane protein. Science. 272:1339-1342.

9. Tsiokas, L., Kim, E., Arnould, T., Sukhatme, V.P., and Walz, G. 1997. Homo- and heterodimeric interactions between the gene products of PKD1 and PKD2. Proc. Natl. Acad. Sci. USA. 94:6965-6970.

10. Qian, F., et al. 1997. PKD1 interacts with PKD2 through a probable coiled-coil domain. Nat. Genet. 16:179-183.

11. Hanaoka, K., et al. 2000. Co-assembly of polycystin-1 and -2 produces unique cation-permeable currents. Nature. 408:990-994.

12. Lu, W., et al. 1997. Perinatal lethality with kidney and pancreas defects in mice with a targeted Pkd1 mutation. Nat. Genet. 17:179-181.

13. Lu, W., et al. 1999. Late onset of renal and hepatic cysts in Pkd1-targeted heterozygotes. Nat. Genet. 21:160-161.

14. Wu, G., et al. 1998. Somatic inactivation of $\mathrm{Pkd} 2$ results in polycystic kidney disease. Cell. 93:177-188.

15. Wu, G., et al. 2000. Cardiac defects and renal failure in mice with targeted mutations in Pkd2. Nat. Genet. 24:75-78.

16. Kim, K., Drummond, I., Ibraghimov-Beskrovnaya, O., Klinger, K., and Arnaout, M.A. 2000. Polycystin 1 is required for the structural integrity of blood vessels. Proc. Natl. Acad. Sci. USA. 97:1731-1736.

17. Boletta, A., et al. 2000. Polycystin-1, the gene product of PKD1, induces resistance to apoptosis and spontaneous tubulogenesis in MDCK cells. Mol. Cell. 6:1267-1273.

18. Rauchman, M.I., Nigam, S.K., Delpire, E., and Gullans, S.R. 1993. An osmotically tolerant inner medullary collecting duct cell line from an SV40 transgenic mouse. Am. J. Physiol. 265:F416-F424.

19. Cantley, L.G., Barros, E.J., Gandhi, M., Rauchman, M., and Nigam, S.K. 1994. Regulation of mitogenesis, motogenesis, and tubulogenesis by hepatocyte growth factor in renal collecting duct cells. Am. J. Physiol. 267:F271-F280.

20. Derman, M.P., Cunha, M.J., Barros, E.J., Nigam, S.K., and Cantley, L.G. 1995. HGF-mediated chemotaxis and tubulogenesis require activation of the phosphatidylinositol 3-kinase. Am. J. Physiol. 268:F1211-F1217.

21. Kjelsberg, C., et al. 1997. Met -/- kidneys express epithelial cells that chemotax and form tubules in response to EGF receptor ligands. Am.J. Physiol. 272:F222-F228.

22. Kim, E., et al. 1999. Interaction between RGS7 and polycystin. Proc. Natl. Acad. Sci. USA. 96:6371-6376.

23. Arnould, T., et al. 1999. Cellular activation triggered by the autosomal dominant polycystic kidney disease gene product PKD2. Mol. Cell. Biol. 19:3423-3434.

24. Ory, D.S., Neugeboren, B.A., and Mulligan, R.C. 1996. A stable humanderived packaging cell line for production of high titer retrovirus/vesicular stomatitis virus G pseudotypes. Proc. Natl. Acad. Sci. USA. 93:11400-11406.

25. Karihaloo, A., O’Rourke, D.A., Nickel, C., Spokes, K., and Cantley, L.G. 2001. Differential MAPK pathways utilized for HGF- and EGF-dependent renal epithelial morphogenesis. J. Biol. Chem. 276:9166-9173.

26. Orellana, S.A., and Avner, E.D. 1998. Cell and molecular biology of kidney development. Semin. Nephrol. 18:233-243.

27. Lupas, A., Van Dyke, M., and Stock, J. 1991. Predicting coiled coils from protein sequences. Science. 252:1162-1164.

28. Qatsha, K.A., Rudolph, C., Marme, D., Schachtele, C., and May, W.S. 1993. Go 6976, a selective inhibitor of protein kinase C, is a potent antagonist of human immunodeficiency virus 1 induction from latent/low-level-producing reservoir cells in vitro. Proc. Natl. Acad. Sci. USA. 90:4674-4678.

29. van Adelsberg, J. 1999. Peptides from the PKD repeats of polycystin, the PKD1 gene product, modulate pattern formation in the developing kidney. Dev. Genet. 24:299-308.

30. Furge, K.A., Zhang, Y.W., and Vande Woude, G.F. 2000. Met receptor tyrosine kinase: enhanced signaling through adapter proteins. Oncogene. 19:5582-5589. 